\title{
NGS approach for new ovarian cancer biomarker discovery Ovarian cancer biomarker discovery
}

\author{
Stefania Brandini ${ }^{1 *}$, Arianna Consiglio ${ }^{1}$, Flavio Licciulli ${ }^{1}$, Sabino Liuni ${ }^{1}$, Anna Napoli ${ }^{2}$, Eugenio Maiorano ${ }^{2}$, Leonardo Resta ${ }^{2}$ and Elda \\ Perlino $^{1}$ \\ ${ }^{1}$ Institute of Biomedical Technologies - National Research Council (ITB-CNR), Italy \\ ${ }^{2}$ Department of Emergency and Organ Transplantation, Operating Unit of Pathological Anatomy, 'Aldo Moro' University, Italy
}

\begin{abstract}
Ovarian cancer is the sixth most common cancer in women and is called 'the silent killer' as most women are not diagnosed until the cancer has already spread, thus resulting in low chances of survival. Given the complex and heterogeneous nature of this neoplasm, it is crucial the identification of molecular biomarkers that might be used for focused and efficient diagnosis, prognosis and therapy.

The main goal of this study was to identify novel biomarkers such as micro RNAs (miRNAs) and their target genes involved in functional pathways associated with ovarian cancer to be used for clinical diagnosis and/or prognosis. For this purpose, we investigated miRNA and mRNA expression in the tissues from ovarian cancer patients using a HT-NGS approach followed by an integrated bioinformatics and biostatistics analysis.
\end{abstract}

\begin{abstract}
Abbreviations: HT-NGS: High Throughput Next Generation Sequencing, FFPE: Formalin-Fixed Paraffin-Embedded, FDR: False Discovery Rate, DEG: Differentially Expressed Gene, GO: Gene Ontology
\end{abstract}

\section{Opinion}

Ovarian cancer is the sixth most common cancer in women and, due to its typically late diagnosis, the most lethal gynecologic malignancy [1]. The American Cancer Society estimates over twenty-two thousand cases of ovarian cancer diagnosed and about fourteen thousand women died of the disease each year [2]. Although traditionally referred to as a single entity, basing on histopathology and moleculargenetic alterations, ovarian carcinomas are subdivided into five main types, essentially considered as distinct diseases, primarily classified according to cell type into serous, mucinous, endometrioid, clear-cell and transitional each with different morphology and biologic behaviour [3]. There is currently no reliable test to diagnose ovarian cancer before symptoms emerge. Given the complex and heterogeneous nature of this neoplasm and the lack of an effective early detection method for it, it is crucial the identification of molecular biomarkers that might be used for a focused and efficient diagnosis, prognosis and therapy. Preliminary studies have suggested that circulating microRNA profiles are altered in women with ovarian cancer and their target gene expression profiles and genetic aberrations [4]. The key role in various biological processes that appear in the early stages of ovarian cancer, such as development, proliferation, differentiation, apoptosis and metastasis, makes miRNA profiles and their target genes good subject matters for the detection of early-stage ovarian cancer signatures [5].

In order to contribute to the identification of miRNA profile for diagnostic molecular biomarkers in the ovarian cancer, we carried out a deep sequencing allowing the simultaneous testing of coding and non-coding RNAs from samples belonging to the most representative histological types of ovarian, serous and endometrioid carcinomas.
In detail, the study was conducted on 21 tissue samples of human ovary FFPE: 12 from carcinoma samples (6 FFPE high-grade serous, 3 FFPE low-grade serous and 3 FFPE endometrioid) and 9 controls (3 FFPE healthy tuba, 3 FFPE serous cistoadenofibroma and 3 FFPE endometriosis). RNAs were extracted using the miRNeasy FFPE Kit (Qiagen) protocol and RNA samples were subjected to massive sequencing on HiSeq2500 Illumina platform using the following protocols respectively on miRNA and mRNA libraries:

- TruSeq Small RNA libraries: Single -reads, 1x50 cycles, 30 million reads / sample;

- TruSeq Stranded Total RNA libraries: paired- end, 2x100 cycles, 30 million reads / sample.

As reported in table 1, a total of 13,682 genes were expressed in the analysed samples. Differentially Expressed Genes (DEGs) were identified based on comparison between tumor versus matched normal tissues using the following statistical thresholds: FDR $<0.05$, mean read count $>100$ and absolute $\log 2$ Fold Change $(|\log 2 \mathrm{FC}|)>1$. Five hundred ninety-eight genes were defined as DEGs, accounted for $\sim 4 \%$ of all expressed genes. Among these, 283 were over-expressed in tumor samples $(\log 2 \mathrm{FC} \geq 1)$, and 315 were under-expressed $(\log 2 \mathrm{FC} \leq-1)$.

DAVID Functional Annotation was used to investigate in which biological pathways and processes the DEG genes are involved, in all analysed samples. Three GO terms, including cell adhesion, extracellular matrix organization, signal transduction, were identified

Correspondence to: Stefania Brandini, Institute of Biomedical Technologies National Research Council (ITB-CNR), Bari, Italy, Tel: +39 0805929673, E-mail brandini.stefania@gmail.com

Key words: ovarian cancer, miRNAs, molecular biomarkers, NGS analysis

Received: April 02, 2018; Accepted: April 14, 2018; Published: April 18, 2018 
Table 1. Sequencing results

\begin{tabular}{|c|c|c|c|}
\hline $\begin{array}{c}\text { Total genes } \\
\text { considered }\end{array}$ & DEGs1 & $\begin{array}{c}\text { Over-expressed } \\
\text { genes }\end{array}$ & $\begin{array}{c}\text { Under-expressed } \\
\text { genes }\end{array}$ \\
\hline 13682 & 598 & 283 & 315 \\
\hline $\begin{array}{c}\text { Total miRNAs } \\
\text { considered }\end{array}$ & $\begin{array}{c}\text { Differentially } \\
\text { expressed miRNAs1 }\end{array}$ & $\begin{array}{c}\text { Over-expressed } \\
\text { miRNAs }\end{array}$ & $\begin{array}{c}\text { Under-expressed } \\
\text { miRNAs }\end{array}$ \\
\hline 2,072 & 85 & 43 & 42 \\
\hline
\end{tabular}

$\mathrm{FDR}<0.05$, mean read count $>100,|\log 2 \mathrm{FC}|>1$

from Biological Process analysis enrichment, thirteen from Cellular Components set and five in the Molecular Function GO set. The GO terms were declared as significantly enriched if showing FDR $<=0.01$.

A complementary differential expression analysis was conducted on miRNAs using an in-house bioinformatics pipeline including mideep2, bowtie, edgeR and DEseq2 software, which highlighted 46 differentially expressed miRNAs between tumor versus matched normal tissues. These noncoding RNAs were compared with those reported in OncomiR (www.oncomir.org), an online resource for exploring miRNA dysregulation in cancer that uses the combined miRNA-seq, RNA-seq and clinical data from The Cancer Genome Atlas [6]. This comparison resulted in the detection, in our sample, of 69 miRNAs already reported as involved in ovarian cancer (32 overand 37 under-expressed) and 19 ( 14 over- and 5 under-expressed) new miRNAs that may be likely associated with ovarian carcinoma.
This original strategy of contemporary miRNA/mRNA analysis may contribute to elucidate the molecular mechanisms responsible for the malignant cell transformation, suggesting further molecular investigations useful to also characterize potential new biomarkers to be used for the ovarian cancer clinical diagnosis and/or prognosis.

\section{References}

1. Pepke S, Ver Steeg G (2017) Comprehensive discovery of subsample gene expression components by information explanation: therapeutic implications in cancer. BMC Med Genomics 10: 12. [Crossref]

2. Siegel RL, Miller KD, Jemal A (2018) Cancer statistics, 2018. CA Cancer J Clin 68: 7-30.

3. Prat J (2012) Ovarian carcinomas: five distinct diseases with different origins, genetic alterations, and clinicopathological features. Virchows Arch 460: 237-249. [Crossref]

4. Lee H, Park CS, Deftereos G, Morihara J, Stern JE, et al. (2012) MicroRNA expression in ovarian carcinoma and its correlation with clinicopathological features. World J Surg Oncol 10: 174. [Crossref]

5. Wang ZH, Xu CJ (2015) Research Progress of MicroRNA in Early Detection of Ovarian Cancer. Chin Med J 128: 3363-3370. [Crossref]

6. Wong NW, Chen Y, Chen S, Wang X, Valencia A (2017) OncomiR: an online resource for exploring pan-cancer microRNA dysregulation. Bioinformatics. 34: 713-715. [Crossref]

Copyright: (C2018 Brandini S. This is an open-access article distributed under the terms of the Creative Commons Attribution License, which permits unrestricted use, distribution, and reproduction in any medium, provided the original author and source are credited. 